\title{
Verification by Behaviour Abstraction
}

\section{A Case Study of Service Interaction Detection in Intelligent Telephone Networks}

\author{
Carla Capellmann ${ }^{a}$, Ralph Demant ${ }^{b}$, Farhad Fatahi-Vanani ${ }^{b}$, \\ Rafael Galvez-Estrada ${ }^{b}$, Ulrich Nitsche ${ }^{b}$ and Peter Ochsenschläger ${ }^{b}$ \\ ${ }^{a}$ Deutsche Telekom, Technologiezentrum Darmstadt \\ Research Group for Functional Aspects of Networks \\ Am Kavalleriesand 3, D-64295 Darmstadt, Germany. \\ Email: carla@fz.telekom.de \\ ${ }^{b}$ GMD - German National Research Centre for Information Technology \\ Institute for Telecooperation Technology \\ Rheinstraße 75, D-64295 Darmstadt, Germany. \\ Email: \{demant,fatahi,galvez, nitsche, ochsenschlaeger\}@darmstadt.gmd.de
}

\section{Introduction}

Modern telephone systems (Intelligent Networks; IN) allow to flexibly introduce new services in the network. There, one is increasingly confronted with undesired interactions of services and service features. The problem of finding service interactions is referred to as service interaction detection (or: feature interaction detection). The aim of the project presented in this abstract is the detection of service interactions on the specification level. The core of such a specification is the so called Basic Call State Model (BCSM) [Q.1], where the services are coupled to.

We specify services and the BCSM using Product Nets [OP95] (high level Petri Nets). By a complete reachability analysis we obtain an automata representation of the complete behaviour of the services. To avoid state-space explosion, a compositional analysis technique can be used [Och95]. To check for service interactions we check temporal properties of the behaviour. In general, the behaviour of the specified services, including the BCSM, is too complex to efficiently check temporal logic formulae on the automata representation of the behaviour (the reachability graph) [CMR93]. Therefore, we first compute an abstraction of the behaviour that has a sufficiently small automata representation to check temporal properties efficiently. When calculating the abstract behaviour we have to be careful not to hide important behaviour with respect to possible interactions of the specified services. As abstraction technique, we use language homomorphisms applied to the language accepted by the automaton representing the behaviour of the combined services. Because it is very important for a telephone system to be able to make progress (we always want to be able to make a call eventually), an important class of properties we have to check in regard to service interaction detection is the class of liveness properties. It is 
especially this class of properties that is very delicate to handle with respect to abstractions.

In the following section we present two example services, Call Forwarding Unconditional (CFU) and Selective Call Rejection (SCR). By discussing these two services we give a brief introduction to the methods we use to detect service interactions. Finally we summarize the current state of our project and give an outlook on the topics we address next.

\section{CFU and SCR}

As a small example to illustrate the basic idea of our approach towards interaction detection, we have selected two simple services, Call Forwarding Unconditional (CFU) and Selective Call Rejection (SCR). CFU is a service that, when active, forwards an incoming call to a selected recepient. Selective Call Rejection (SCR) prohibits an incoming call to be signalled; i.e. an incoming call is rejected without notifying the intended receiver.

To keep a first model of CFU and SCR simple, we describe both services on a high abstraction level, assuming a very simplified basic call process where the activation of a service simply depends on its parameters: The parameter that activates CFU is the telephone number to which an incoming call has to be forwarded. SCR's parameter is a list of telephone numbers such that calls by a caller whose number is in the list are rejected.

\section{Interaction of CFU and SCR}

As already mentioned, we have specified CFU and SCR logically as a Product Net. Here, "logically" means that we did not involve the underlying model of the telephone system, the Basic Call State Model (BCSM) as defined in [Q.1]. This first step was intended to show that the methods we propose for service interaction detection are indeed suitable for this task.

We analyzed our model of CFU and SCR for all combinations of CFU and SCR being active and being not active involving 3 subscribers (users). The complete reachability analysis of each active/not active combination of CFU and SCR led to automata representations having at most 780 states. All the automata were deadlock free. This is an important property for the specified services; formally this is a safety property. As said in the introduction, the properties that guarantee progress are the liveness properties. The liveness property we want to check is whether it is always possible to eventually get a connection to an intended receiver. If connect is the event that describes the establishment of a desired connection, then in temporal logic we want to check if the formulae $\square \diamond$ connect ("always eventually connect") is a liveness property with respect to the behaviour of the specified services. We can check this property on an abstraction of the behaviour that keeps the connect event visible and hides all other events. Formally, such an abstraction is defined by a language homomorphism. 
To be able to check liveness properties on the abstraction[Nit94a, Nit94b, NO95], the homomorphisms have to satisfy a special condition called simplicity of language homomorphisms [Och94].

Applying a suitable simple homomorphism to the automata representation of the behaviour of the specification of CFU and SCR leads to an empty automaton, if CFU and SCR are active such that the number of the caller is in the rejection list of the subscriber to whom the call is forwarded. That means, for this constellation, $\square \bigcirc$ connect is not a property of the specification, even if the originally called subscriber did not want to reject that call. Therefore, CFU and SCR interact by SCR interfering CFU.

\section{Verification of the BCSM}

So far, we only have checked the interaction of services on a logical level. Nevertheless, for detecting service interactions in a more realistic specification, the underlying telephone system (BCSM) has to be specified as well, to embed the services in it. So we have already specified the BCSM as a Product Net and have verified its correctness. More precisely, using simple homomorphisms, we were able to prove that our specification shows exactly the behaviour as it is described by the automata in [Q.1].

\section{Conclusion}

As shown above, verification of temporal properties on behaviour abstractions is suitable for detecting service interactions. In the example that we briefly explained the abstraction step was not really necessary, for the example itself is small enough to check temporal properties directly on the behaviour.

A complete analysis of the BCSM model already leads to a behaviour representation having several thousand states. Including services, we expect several tenthousand states. This reaches an order of magnitude of the number of states that makes abstraction techniques absolutely necessary. Here, even the analysis of the specification can become difficult with respect to complexity. Hence, a compositional analysis technique of specifications that allows to compute a representation of an abstract behaviour without exhaustive construction of the complete state space is currently developed [Och95]. We expect that our approach will allow us to check several combinations of services for undesired interactions where any direct verification approach without abstractions would be intractable. 


\section{References}

[CMR93] Pierre Combes, Max Michel, and Béatrice Renard. Formal verification of telecommunication service interactions using SDL methods and tools. In O. Faergemand and A. Sarma, editors, SDL'93: Using Objects, pages 441452. Elsevier, 1993.

[Nit94a] Ulrich Nitsche. Propositional linear temporal logic and language homomorphisms. In Anil Nerode and Yuri V. Matiyasevich, editors, Logical Foundations of Computer Science '94-Logic at St. Petersburg, volume 813 of Lecture Notes in Computer Science, pages 265-277. Springer Verlag, 1994.

[Nit94b] Ulrich Nitsche. A verification method based on homomorphic model abstraction. In Proceedings of the 13th Annual ACM Symposium on Principles of Distributed Computing, page 393, Los Angeles, 1994. ACM Press.

[NO95] Ulrich Nitsche and Peter Ochsenschläger. Approximately satisfied properties of systems and simple language homomorphisms. Arbeitspapiere der GMD 965, Gesellschaft für Mathematik und Datenverarbeitung (GMD), Darmstadt, December 1995.

[Och94] Peter Ochsenschläger. Verification of cooperating systems by simple homomorphisms using the product net machine. In Jörg Desel, Andreas Oberweis, and Wolfgang Reisig, editors, Workshop: Algorithmen und Werkzeuge für Petrinetze, pages 48-53. Humboldt Universität Berlin, 1994.

[Och95] Peter Ochsenschläger. Compositional verification of cooperating systems using simple homomorphisms. In Jörg Desel, Hans Fleischhack, Andreas Oberweis, and Sonnenschein Michael, editors, Workshop: Algorithmen und Werkzeuge für Petrinetze, pages 8-13. Universität Oldenburg, 1995.

[OP95] Peter Ochsenschläger and Rainer Prinoth. Modellierung verteilter Systeme - Konzeption, Formale Spezifikation und Verifikation mit Produktnetzen. Vieweg, Wiesbaden, 1995.

[Q.1] Draft Revised ITU-T Recommendation Q.1214: Distributed Functional Plane for Intelligent Network CS-1. March 1995. 